\title{
Middle East Respiratory Syndrome (MERS) coronavirus seroprevalence in domestic livestock in Saudi Arabia, 2010 to 2013
}

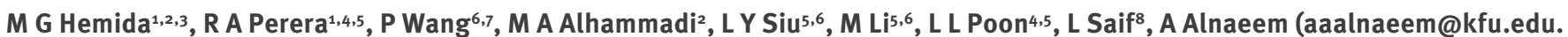
sa)', M Peiris (malik@hku.hk)4,5,6

1. These authors contributed equally to this work

2. Department of Microbiology and Parasitology, College of Veterinary Medicine, King Faisal University, Saudi Arabia

3. Department of Virology, Faculty of Veterinary Medicine, Kaferelsheik University, Egypt

4. Centre of Influenza Research, The University of Hong Kong, Hong Kong SAR, China

5. School of Public Health, The University of Hong Kong, Hong Kong SAR, China

6. HKU-Pasteur Pole, The University of Hong Kong, Hong Kong SAR, China

7. Key Laboratory of Protein and Peptide Pharmaceuticals, Chinese Academy of Sciences - University of Tokyo Joint Laboratory of Structural Virology and Immunology, Beijing, China

8. Food Animal Health Research Program, Veterinary Preventive Medicine Department, OARDC/The Ohio State University, Wooster, United States of America

9. Department of Clinical Studies, College of Veterinary Medicine, King Faisal University, Saudi Arabia

Citation style for this article:

Hemida MG, Perera RA, Wang P, Alhammadi MA, Siu LY, Li M, Poon LL, Saif L, Alnaeem A, Peiris M. Middle East Respiratory Syndrome (MERS) coronavirus seroprevalence in domestic livestock in Saudi Arabia, 2010 to 2013. Euro Surveill. 2013;18(50):pii=20659. Available online: http://www.eurosurveillance.org/ ViewArticle.aspx?Articleld $=\mathbf{2 0 6 5 9}$

Article submitted on 04 December 2013 / published on 12 December 2013

In Saudi Arabia, including regions of Riyadh and Al Ahsa, pseudoparticle neutralisation (ppNT) and microneutralisation (MNT) tests detected no antibodies to Middle East Respiratory Syndrome coronavirus (MERS-CoV) in sheep $(n=100)$, goats $(n=45)$, cattle $(n=50)$ and chickens $(n=240)$. Dromedary camels however, had a high prevalence of MERS-CoV antibodies. Bovine coronavirus (BCoV) infected sera from cattle had no cross-reactivity in MERS-CoV ppNT or MNT, while many dromedary camels' sera reacted to both BCoV and MERS-CoV. Some nevertheless displayed specific serologic reaction profiles to MERS-CoV.

In a seroepidemiological study of domestic livestock (sheep, goats, cattle, chicken) and dromedary camels from Saudi Arabia, we find that only dromedary camels have evidence of seropositivity to Middle East Respiratory Syndrome coronavirus (MERS-CoV), suggesting an infection with a MERS-CoV-like virus. Although some dromedary camels examined had specific serologic reaction profiles to MERS-CoV, many had sera displaying reactivity to both MERS-CoV and bovine coronavirus ( $\mathrm{BCoV}$ ) and the sera appear to have an unusually broad pattern of cross-reactivity for these related viruses. This needs to be considered when interpreting seroepidemiological data by carrying out parallel microneutralisation tests for both MERS CoV and $\mathrm{BCoV}$.

\section{Middle East Respiratory}

\section{Syndrome coronavirus}

MERS coronavirus was recognised as a cause of severe human respiratory disease in 2012 [1]. As of 22 November 2013, 157 laboratory-confirmed, and 19 other probable cases of MERS have been reported, 69 of these being fatal [2]. Sporadic or index cases account for approximately $40 \%$ of the cases and have occurred in Jordan, Kuwait, Oman, Qatar, Saudi Arabia and United Arab Emirates. Imported cases have been reported in France, Germany, Italy, Spain, Tunisia and United Kingdom, with secondary transmission documented in some of these cases [2,3]. Human-to-human transmission has occasionally been observed, the largest cluster of such secondary transmission being reported from Al Ahsa, Saudi Arabia [4]. Phylogenetic analysis suggests considerable diversity within MERS$\mathrm{CoV}$ sequences analysed to date, with two distinct clades of virus being noted [5].

Index cases of MERS appear to be zoonotic in origin, with contact with domestic livestock (e.g. camels, sheep) being reported in some of these patients [3]. But neither the proximate animal source of human infection nor the natural reservoir of the virus is known. Closely related, but not identical viruses have been found in species of insectivorous bats [6]. There is a preliminary report of a short fragment of virus genome almost identical to MERS-CoV reportedly found in Egyptian tomb bats [7].

Two recent studies from Oman and Egypt respectively, two Middle-Eastern countries adjacent to affected ones, reported high rates of MERS-CoV seroprevalence in dromedary camels $[8,9]$. However, there have been no seroepidemiological data of domestic livestock, camels or wildlife from affected countries, to date. 
Locations where serum samples were collected for the Middle East Respiratory Syndrome (MERS) coronavirus seroepidemiological study in domestic livestock, Saudi Arabia, 2010-2013

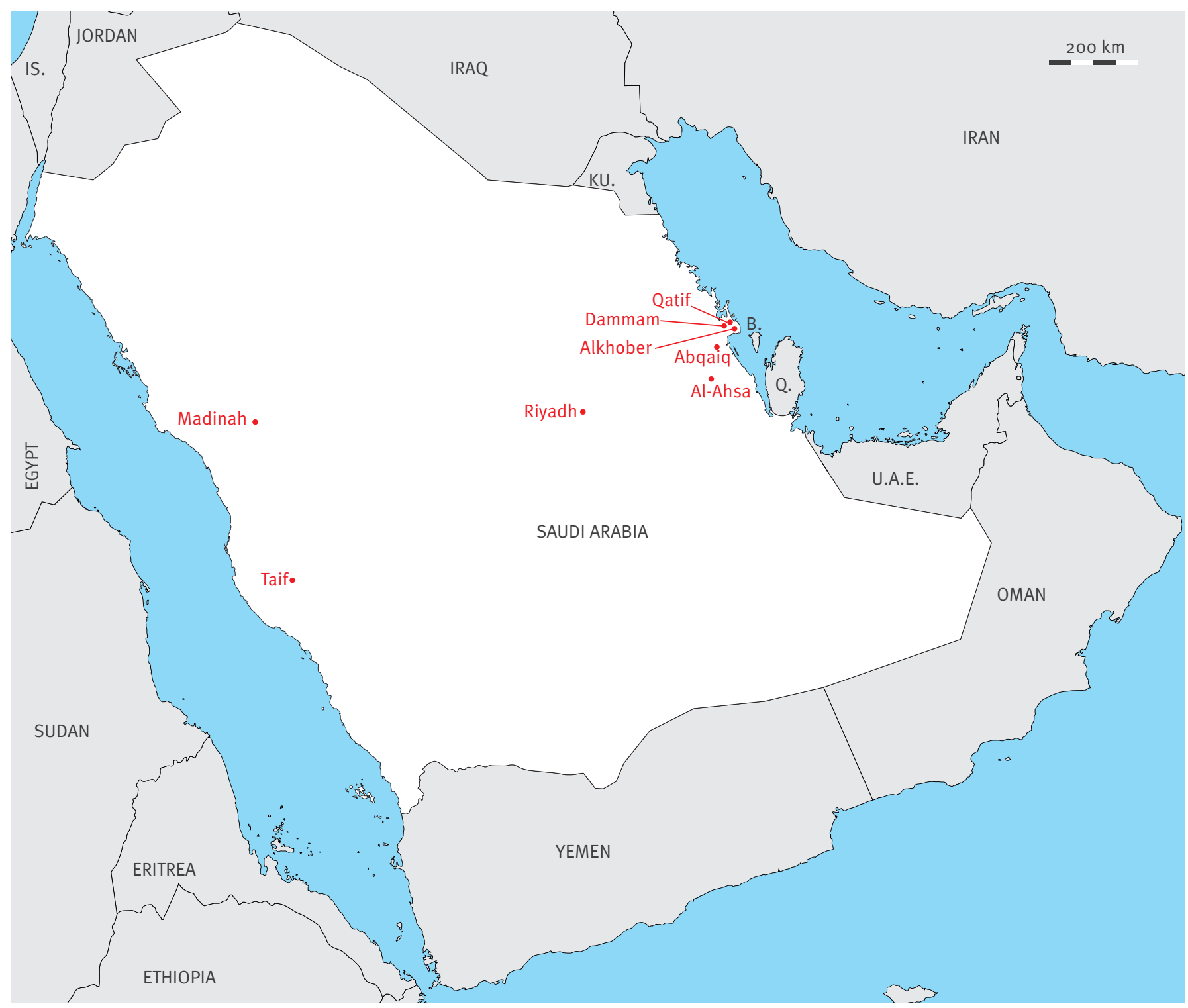

\section{Seroepidemiological study}

Serum samples were collected from dromedary camels $(n=310)$, sheep $(n=100)$, goats $(n=45)$, cattle $(n=50)$ and chicken $(n=240)$ from Riyadh, Al Ahsa and other regions of Saudi Arabia (Figure).

The MERS-CoV serology methods used have been described in a previous publication [9]. The sera were heat inactivated at $56^{\circ} \mathrm{C}$ for 30 minutes and screened for antibody to MERS-CoV at a serum dilution of 1:20 in a MERS-CoV pseudoparticle neutralisation test (ppNT) [9]. Camel sera $(n=56)$ that were seropositive at a screening dilution of 1:20 in the ppNT assay were randomly selected, representing each age group, less than one year $(n=15)$, one to three years $(n=12)$, four to five years $(n=16)$ and older than five years $(n=13)$, and titrated to end point in the MERS-CoV ppNT, MERS-CoV microneutralisation test (MNT) and bovine coronavirus (BCoV) MNT.

The MNTs were done using MERS-CoV (strain: EMC) obtained from Dr R Fouchier, Erasmus MC, Rotterdam and bovine coronavirus (ATCC BRCV-OK-0514-2). The MNTs were done on Vero cells (ATCC CCL-81) for MERSCoV and HRT-18G cells (obtained from ATCC) for BCoV, respectively. Serial two-fold dilutions of heat-inactivated sera ( $56^{\circ} \mathrm{C}$ for 30 minutes) were mixed with equal volumes of 200 tissue culture infective dose (TCID) $)_{50}$ of virus and incubated for one hour at $37^{\circ} \mathrm{C}$. The virus-serum mixture was then added in quadruplicate to cell monolayers in 96-well microtitre plates. After one hour of adsorption, the virus-serum mixture 
Location and time of sampling of animals chosen for MERS-CoV ppNT and proportion of serum samples positive at 1:20 dilution, Saudi Arabia, 2010-2013 (n=745 samples)

\begin{tabular}{|l|c|c|c|c|}
\hline Animals & Location (number of animals) & Collection period & $\begin{array}{c}\text { Serum samples collected } \\
\text { N }\end{array}$ & $\begin{array}{c}\text { Sera positive in MERS-CoV } \\
\text { ppNT N (\%) }\end{array}$ \\
\hline Dromedary camels & Al-Ahsa (120), Riyadh (190) & $2012-2013$ & 310 & $280(90)$ \\
\hline Sheep & Al-Ahsa (100) & $2012-2013$ & 100 & 0 (0) \\
\hline Goats & $\begin{array}{c}\text { Al-Ahsa (15), Taif (10), Madinah } \\
(10), \text { Qatif (10) }\end{array}$ & $2010-2012$ & 45 & $0(0)$ \\
\hline Chicken & $\begin{array}{c}\text { Al-Ahsa (120), Dammam and } \\
\text { Alkhober (80), Abqaiq (40) }\end{array}$ & $2012-2013$ & 240 & $0(0)$ \\
\hline Cattle & $\begin{array}{c}\text { Al-Ahsa (17), Taif (13), Madinah } \\
(10), \text { Qatif (10) }\end{array}$ & $2010-2013$ & 50 & $0(0)$ \\
\hline
\end{tabular}

MERS-CoV ppNT: Middle East Respiratory Syndrome pseudoparticle neutralisation test.

Riyadh province is in central Saudi Arabia; Al Ahsa, Quatif, Dammam, Alkhober and Abqaiq are in the eastern part of Saudi Arabia; Madinah and Taif are in the western part of Saudi Arabia (Figure).

was removed and $150 \mu$ of fresh culture medium was added to each well and the plates incubated at $37^{\circ} \mathrm{C}$ in $5 \% \mathrm{CO}_{2}$ in a humidified incubator. A virus back-titration was performed without immune serum to assess input virus dose.

Cytopathic effect (CPE) was read at three days post infection for MERS-CoV and four days post infection for $\mathrm{BCoV}$. The highest serum dilution that completely protected the cells from CPE in half of the wells was defined as the neutralising antibody titre. Positive and negative control sera were included in each assay.

As positive controls, we used a convalescent serum from a human patient with MERS kindly provided by Dr C Drosten, Institute of Virology, University of Bonn Medical Centre, Bonn (MERS-CoV ppNT 1:160); and sera from two experimentally infected macaques (ppNT titres 1:40) and a non-infected control macaque (ppNT «1:20) kindly provided by Bart Haagmans, Erasmus MC as reported previously [9]. Additional positive and negative controls used were sera from dromedary camels from Egypt previously found to be seropositive (ppNT 1:320) and sero-negative (ppNT<1:20) [9]. Antisera to $\mathrm{BCoV}$ from an experimentally inoculated gnotobiotic calf and guinea pig were provided by Dr. Linda Saif.

\section{Results}

Sera that were positive in the MERS-CoV ppNT test at a screening dilution of 1:20 are shown in Table 1.

A gnotobiotic calf and guinea pig B CoV antisera that had a homologous reaction titre of $\geq 1: 1,280$ and $1: 80$ respectively, did not cross-neutralise MERS-CoV in either the MERS-CoV ppNT or MNT. None of the sheep, goat, cattle or chicken sera had any MERS-CoV ppNT

\section{TABLE 2}

Stratification by age and location of dromedary camels testing positive in the MERS-CoV ppNT assay at a serum dilution of 1:20, Saudi Arabia, 2012-2013 ( $\mathrm{n}=310$ animals)

\begin{tabular}{|l|c|c|c|c|c|}
\hline \multirow{2}{*}{$\begin{array}{l}\text { Age } \\
\text { group } \\
\text { (years) }\end{array}$} & $\begin{array}{c}\text { Animals tested } \\
\mathrm{N}\end{array}$ & $\begin{array}{c}\text { Animals testing } \\
\text { positive } \\
\mathrm{N}(\%)\end{array}$ & $\begin{array}{c}\text { Animals tested } \\
\mathrm{N}\end{array}$ & $\begin{array}{c}\text { Animals testing } \\
\text { positive } \\
\mathrm{N}(\%)\end{array}$ & $\begin{array}{c}\text { Animals tested } \\
\mathrm{N}\end{array}$ \\
\hline$<1$ & 21 & $18(86)$ & 44 & $29(66)$ & $\begin{array}{c}\text { Animals testing } \\
\text { positive } \\
\mathrm{N}(\%)\end{array}$ \\
\hline $1-3$ & 55 & $52(94)$ & 51 & $49(96)$ & 65 \\
\hline $4-5$ & 52 & $51(98)$ & 24 & $23(96)$ & 106 \\
\hline$>5$ & 62 & $57(92)$ & 1 & $101(95)$ & 76 \\
\hline Total & 190 & $178(94)$ & 120 & $102(85)$ & 63 \\
\hline
\end{tabular}


TABLE 3A

Serology titres for MERS-CoV and BCoV in dromedary camel sera, Saudi Arabia, 2012-2013 (n=56)

\begin{tabular}{|c|c|c|c|c|c|c|}
\hline Sera IDs & $\begin{array}{l}\text { Age group in } \\
\text { years }\end{array}$ & MERS-CoV ppNT titre & MERS-CoV MNT titre & BCoV MNT titre & Reaction profile & Region \\
\hline 1 & $\ll 1$ & $1: 2,560$ & $1: 160$ & $1: 20$ & MERS-CoV specific & Riyadh \\
\hline 2 & $\ll 1$ & $1: 320$ & $1: 20$ & $<1: 10$ & MERS-CoV specific & Riyadh \\
\hline 3 & $\ll 1$ & $\geq 1: 5,120$ & $1: 640$ & $1: 40$ & MERS-CoV specific & Al-Ahsa \\
\hline 4 & $\ll 1$ & $1: 1,280$ & $1: 320$ & $<1: 10$ & MERS-CoV specific & Al-Ahsa \\
\hline 5 & $\ll 1$ & $1: 640$ & $1: 40$ & $1: 160$ & BCoV specific & Riyadh \\
\hline 6 & $\ll 1$ & $1: 640$ & $1: 40$ & $1: 320$ & BCoV specific & Riyadh \\
\hline 7 & $\langle 1$ & $1: 160$ & $1: 10$ & $1: 40$ & BCoV specific & Riyadh \\
\hline 8 & $\ll 1$ & $1: 1,280$ & $1: 80$ & $1: 640$ & BCoV specific & Al-Ahsa \\
\hline 9 & $\ll 1$ & $\langle 1: 20$ & $<1: 10$ & $1: 160$ & BCoV specific & Al-Ahsa \\
\hline 10 & $\ll 1$ & $1: 80$ & $1: 10$ & $1: 40$ & BCoV specific & Al-Ahsa \\
\hline 11 & $\ll 1$ & $1: 80$ & $1: 10$ & $<1: 10$ & Indeterminate & Riyadh \\
\hline 12 & $\ll 1$ & $\geq 1: 5,120$ & $1: 640$ & $1: 640$ & Indeterminate & Riyadh \\
\hline 13 & $\ll 1$ & $1: 2,560$ & $1: 320$ & $1: 320$ & Indeterminate & Al-Ahsa \\
\hline 14 & $\ll 1$ & $1: 2,560$ & $1: 320$ & $1: 320$ & Indeterminate & Al-Ahsa \\
\hline 15 & $\ll 1$ & $1: 1,280$ & $1: 160$ & $1: 320$ & Indeterminate & Al-Ahsa \\
\hline 16 & $1-3$ & $\geq 1: 5,120$ & $\geq 1: 1,280$ & $<1: 10$ & MERS-CoV specific & Riyadh \\
\hline 17 & $1-3$ & $1: 160$ & $1: 20$ & $<1: 10$ & MERS-CoV specific & Riyadh \\
\hline 18 & $1-3$ & $\geq 1: 5,120$ & $\geq 1: 1,280$ & $1: 80$ & MERS-CoV specific & Riyadh \\
\hline 19 & $1-3$ & $1: 640$ & $1: 80$ & $<1: 10$ & MERS-CoV specific & Al-Ahsa \\
\hline 20 & $1-3$ & $1: 640$ & $1: 160$ & $<1: 10$ & MERS-CoV specific & Al-Ahsa \\
\hline 21 & $1-3$ & $1: 640$ & $1: 40$ & $1: 160$ & BCoV specific & Riyadh \\
\hline 22 & $1-3$ & $1: 20$ & $<1: 10$ & $1: 160$ & BCoV specific & Riyadh \\
\hline 23 & $1-3$ & $1: 2,560$ & $1: 80$ & $>1: 1,280$ & BCoV specific & Al-Ahsa \\
\hline 24 & $1-3$ & $1: 320$ & $1: 10$ & $1: 20$ & Indeterminate & Riyadh \\
\hline 25 & $1-3$ & $1: 640$ & $1: 80$ & $1: 40$ & Indeterminate & Riyadh \\
\hline 26 & $1-3$ & $1: 160$ & $1: 20$ & $1: 40$ & Indeterminate & Al-Ahsa \\
\hline 27 & $1-3$ & $1: 1,280$ & $1: 640$ & $1: 320$ & Indeterminate & Al-Ahsa \\
\hline 28 & $4-5$ & $\geq 1: 5,120$ & $1: 640$ & 1:160 & MERS-CoV specific & Riyadh \\
\hline 29 & $4-5$ & $1: 160$ & $1: 160$ & $1: 40$ & MERS-CoV specific & Riyadh \\
\hline 30 & $4-5$ & $\geq 1: 5,120$ & $1: 320$ & $1: 80$ & MERS-CoV specific & Riyadh \\
\hline 31 & $4-5$ & $\geq 1: 5,120$ & $1: 640$ & $1: 80$ & MERS-CoV specific & Riyadh \\
\hline 32 & $4-5$ & $\geq 1: 5,120$ & $\geq 1: 1,280$ & $1: 40$ & MERS-CoV specific & Riyadh \\
\hline 33 & $4-5$ & $1: 640$ & $1: 160$ & $<1: 10$ & MERS-CoV specific & Riyadh \\
\hline 34 & $4-5$ & $\geq 1: 5,120$ & $\geq 1: 1,280$ & $\ll 1: 10$ & MERS-CoV specific & Al-Ahsa \\
\hline 35 & $4-5$ & $1: 320$ & $1: 40$ & $1: 640$ & BCoV specific & Riyadh \\
\hline 36 & $4-5$ & $1: 1,280$ & $1: 320$ & $>1: 1280$ & BCoV specific & Riyadh \\
\hline 37 & $4-5$ & $1: 2,560$ & $1: 640$ & $11: 1280$ & BCoV specific & Riyadh \\
\hline 38 & $4-5$ & $1: 320$ & $1: 40$ & $1: 320$ & BCoV specific & Al-Ahsa \\
\hline 39 & $4-5$ & $\geq 1: 5,120$ & 1:320 & $>1: 1,280$ & BCoV specific & Al-Ahsa \\
\hline 40 & $4-5$ & 1:160 & $1: 40$ & $1: 320$ & BCoV specific & Al-Ahsa \\
\hline 41 & $4-5$ & $1: 1,280$ & $1: 160$ & $1: 320$ & Indeterminate & Riyadh \\
\hline 42 & $4-5$ & $1: 2,560$ & $1: 320$ & $1: 640$ & Indeterminate & Riyadh \\
\hline 43 & $4-5$ & $1: 1,280$ & $1: 160$ & $1: 320$ & Indeterminate & Al-Ahsa \\
\hline
\end{tabular}

BCoV: bovine coronavirus; ID: identity; MERS-CoV: Middle East Respiratory Syndrome coronavirus; MNT: microneutralisation test; ppNT: pseudoparticle neutralisation test.

The reaction profile was denoted as MERS-CoV specific if the antibody titre to MERS-CoV was $\geq 4$ fold higher than BCoV; and BCoV specific if the titre to $\mathrm{BCOV}$ was $\geq 4$ fold higher than for MERS-CoV. Other sera are regarded to have an indeterminate reaction profile. 
Serology titres for MERS-CoV and BCoV in dromedary camel sera, Saudi Arabia, 2012-2013 (n=56)

\begin{tabular}{|l|c|c|c|c|c|c|}
\hline Sera IDs & $\begin{array}{c}\text { Age group in } \\
\text { years }\end{array}$ & MERS-CoV ppNT titre & MERS-CoV MNT titre & BCoV MNT titre & Reaction profile & Region \\
\hline 44 & $>5$ & $\geq 1: 5,120$ & $1: 640$ & $1: 80$ & MERS-CoV specific & Riyadh \\
\hline 45 & $>5$ & $1: 1,280$ & $1: 320$ & $1: 80$ & MERS-CoV specific & Riyadh \\
\hline 46 & $>5$ & $1: 640$ & $1: 640$ & $1: 40$ & MERS-CoV specific & Riyadh \\
\hline 47 & $>5$ & $<1: 20$ & $1: 10$ & $1: 80$ & BCoV specific & Riyadh \\
\hline 48 & $>5$ & $1: 80$ & $1: 80$ & $1: 320$ & BCoV specific & Riyadh \\
\hline 49 & $>5$ & $\geq 1: 5,120$ & $1: 640$ & $11: 1280$ & Indeterminate & Riyadh \\
\hline 50 & $>5$ & $1: 1,280$ & $1: 320$ & $1: 160$ & Indeterminate & Riyadh \\
\hline 51 & $>5$ & $1: 1,280$ & $1: 640$ & $1: 320$ & Indeterminate & Riyadh \\
\hline 52 & $>5$ & $\geq 1: 5,120$ & $1: 640$ & $11: 1,280$ & Indeterminate & Riyadh \\
\hline 53 & $>5$ & $1: 1,280$ & $1: 640$ & $1: 320$ & Indeterminate & Riyadh \\
\hline 54 & $>5$ & $1: 640$ & $1: 640$ & $1: 640$ & Indeterminate & Riyadh \\
\hline 55 & $>5$ & $\geq 1: 5,120$ & $\geq 1: 1,280$ & $11: 1,280$ & Indeterminate & Riyadh \\
\hline 56 & $>5$ & $1: 320$ & $1: 20$ & $1: 40$ & Indeterminate & Riyadh \\
\hline
\end{tabular}

BCoV: bovine coronavirus; ID: identity; MERS-CoV: Middle East Respiratory Syndrome coronavirus; MNT: microneutralisation test; ppNT: pseudoparticle neutralisation test.

The reaction profile was denoted as MERS-CoV specific if the antibody titre to MERS-CoV was $\geq 4$ fold higher than $B$ CoV; and BCoV specific if the titre to $\mathrm{BCoV}$ was $\geq 4$ fold higher than for MERS-CoV. Other sera are regarded to have an indeterminate reaction profile.

activity while 280 of 310 (90\%) dromedary camel sera were seropositive. The age-group and location of the camels tested and their serostatus in the screening assay are shown in Table 2. While 47 of $65(72 \%)$ of camels less than one year of age were seropositive, 233 of 245 (95\%) of camels older than one year were seropositive to MERS-CoV in the ppNT test (chi-squared test, p<0.01).

A randomly selected subset of 54 ppNT seropositive sera and two ppNT negative sera were titrated by MERS-CoV ppNT, MERS-CoV MNT and BCoV MNT (Table 3). MERS-CoV ppNT titres ranged from 1:20 to $\geq 1: 5,120$ and MERS-CoV MNT titres ranged from $<1: 10$ (in one serum only) to $\geq 1: 1,280$. Dromedary camel sera, which have $\geq 4$ fold higher antibody MERS-CoV MNT titres compared to BCoV MNT titres are denoted as 'MERSCoV specific' reaction patterns. Sera with comparable (within 4-fold) titres to both viruses and are denoted as 'indeterminate' reaction patterns while those that have $\geq 4$ fold higher titres to $\mathrm{BCoV}$ are regarded as ' $\mathrm{BCoV}$ specific'.

Twelve of the 50 cattle sera were randomly selected for BCoV MNT and 11 sera were positive (Table 4).

\section{Discussion}

This study was conducted Saudi Arabia, including in central and eastern provinces from which most of the human cases of MERS-CoV hitherto had been detected; in particular, the Al Ahsa region from where the largest cluster of human-to-human transmission of the disease has occurred [4] and the Riyadh region, which appears to host the greatest genetic diversity of MERS-CoV within Saudi Arabia [5]. None of the cattle, goat, sheep or chickens had any detectable antibody to MERS-CoV. While larger numbers of such livestock in the vicinity of confirmed cases of MERS need to be examined, our data do not indicate that these species of domestic livestock are commonly infected by MERSCoV. As expected, BCoV antibody was common in cattle with 11 of 12 cattle sera having evidence of $\mathrm{BCoV}$ MNT antibody, but these sera did not cross-react with MERS-CoV.

Dromedary camels from the central province of Riyadh as well as the eastern province, Al Ahsa, had comparably high levels of seropositivity to MERS-CoV by ppNT tests. Camels less than one year of age had lower seroprevalence ( $72 \%)$ than those older than one year of age (95\%) ( $p$ <0.01). Some dromedary camels have high titres to MERS-CoV in the absence of any reactivity to $\mathrm{BCOV}$ confirming that these animals are being infected by a virus very different to $\mathrm{BCoV}$, which could be identical or closely related to MERS-CoV $[8,9]$. Similarly, some animals appear to be infected by a $\mathrm{BCoV}$-like virus without cross-reactivity to MERS-CoV. If we consider just the 'MERS-CoV specific' reactions, we observe that these animals are getting infected within the first year of life (Table 3). 
TABLE 4

Serology titres for MERS-CoV and BCoV in cattle sera, Saudi Arabia, 2010-2013 ( $\mathrm{n}=12$ )

\begin{tabular}{|l|c|c|c|}
\hline Sera ID & $\begin{array}{c}\text { MERS-CoV } \\
\text { ppNT titre }\end{array}$ & $\begin{array}{c}\text { MERS-CoV MNT } \\
\text { titre }\end{array}$ & BCoV MNT titre \\
\hline 1 & $<1: 20$ & $<1: 10$ & $1: 40$ \\
\hline 2 & $<1: 20$ & $<1: 10$ & $<1: 10$ \\
\hline 3 & $<1: 20$ & $<1: 10$ & $1: 640$ \\
\hline 4 & $<1: 20$ & $<1: 10$ & $1: 160$ \\
\hline 5 & $<1: 20$ & $<1: 10$ & $1: 80$ \\
\hline 6 & $<1: 20$ & $<1: 10$ & $1: 40$ \\
\hline 7 & $<1: 20$ & $<1: 10$ & $1: 160$ \\
\hline 8 & $<1: 20$ & $<1: 10$ & $1: 40$ \\
\hline 9 & $<1: 20$ & $<1: 10$ & $1: 40$ \\
\hline 10 & $<1: 20$ & $<1: 10$ & $1: 640$ \\
\hline 11 & $<1: 20$ & $<1: 10$ & $1: 160$ \\
\hline 12 & $<1: 20$ & $<1: 10$ & $1: 160$ \\
\hline
\end{tabular}

BCoV: bovine coronavirus; ID: identity; MERS-CoV: Middle East Respiratory Syndrome coronavirus; MNT: microneutralisation test; ppNT: pseudoparticle neutralisation test.

Naturally infected cattle with BCoV titres of up to $1: 640$ and an experimentally infected calf with a titre of $\geq 1: 1,280$ had no cross-reaction to MERS-CoV in ppNT and MNT assays. Previously, we had shown that human sera with antibody to Severe Acute Respiratory Syndrome (SARS)-CoV failed to react with MERS-CoV, moreover SARS-CoV was not neutralised by dromedary camel sera with high antibody titres to MERS-CoV [9]. In contrast, while some dromedary camel sera had mono-specific reactions to either MERS CoV or BCoV, many sera have 'indeterminate' reaction profiles with reactivity to both MERS-CoV and $\mathrm{BCOV}$ (Table 3 ). It is possible that sequential infection of dromedary camels with different coronaviruses may lead to a broadening of the serological cross-reaction profile. Alternatively, it is possible that the unusual single chain immunoglobulins possessed by camels [10] may give broader serological cross-reactivity than is seen with other species. In any event, these findings highlight the importance of parallel titration of sera to both MERS-CoV and $\mathrm{BCoV}$ when interpreting seroepidemiological results.

The exact identity of this MERS-CoV-like virus can only be established by characterising this virus from dromedary camel specimens. As many of the dromedary camels sampled in this study were already seropositive to MERS-CoV, we did not attempt to detect viral RNA in the serum samples. There are (as yet unpublished) reports of detecting a MERS-CoV-like virus in camel specimens from Saudi Arabia and Qatar [2]. Assuming that MERSCoV seropositivity in MNTs, irrespective of the infecting virus that leads to such seropositivity, correlates with resistance to, or reduced susceptibility to re-infection, these results would suggest that the search for virus in these animals should be focused on dromedary camels «1 year of age. Studies involving follow-up of herds of camels from time of calving though the first year of life with serial blood samples together with oral and rectal or fresh faecal swabs would better define the ecology of the MERS-CoV-like virus infecting these animals and provide virus for genetic characterisation. Such studies are a priority to determine whether dromedary camels are in fact a source of human MERS-CoV infection or whether they are being infected by a ubiquitous novel coronavirus closely related to MERS-CoV. As illustrated with SARS a decade ago and avian influenza $A\left(\mathrm{H}_{7} \mathrm{~N} 9\right)$ recently, identification of the animal source and the setting within which zoonotic transmission occurs can provide options for reducing repeated zoonotic transmissions and enhancing global public health. Finally, given the high titres of neutralising antibody to MERS CoV commonly seen in dromedary camels, serum from these animals may provide an option for passive immunotherapy of patients with MERS, from whom no specific antiviral therapy currently exists.

\section{Acknowledgements}

This study was supported by a grant from the Deanship of Scientific Research at King Faisal University. Work at the University of Hong Kong was supported by grants from the European Community Seventh Framework Program (FP7/2007-2013) under project European management Platform for Emerging and Re-emerging Disease entities (Grant agreement No. 223498) (EMPERIE) and a research contract from the National Institute of Allergy and Infectious Diseases (NIAID) contract HHSN266200700005C

Conflict of interest

None declared.

Authors' contributions

Maged Gomaa Hemida (mhemida@kfu.edu.sa), Malik Peiris (malik @hku.hk) designed, coordinated and supervised the study and wrote the manuscript. Ranawaka APM Perera (mahenp@hku.hk) developed the MERS-CoV and bovine coronavirus microneutralisation tests, carried out the MERS-CoV tests in BSL3 containment and the MERS pseudoparticle assays. Pei-gang Wang (pgwang@hkucc.hku. hk) developed the MERS-CoV pseudotype assay. Mohamed A Alhammadi (malhammadi@kfu.edu.sa): Specimen collection and data recording. Lewis YL Siu (ylsiu@hku.hk) and Ming-yuan Li (Imy288@hku.hk) carried out the MERS-CoV pseudoparticle assays. Leo LLM Poon (Ilmpoon@hku.hk): Provided advice on laboratory methods. Linda Saif (saif.2@ osu.edu): Provided reagents and advised on study design. Abdelmohsen Alnaeem (aaalnaeem@kfu.edu.sa): Designed and supervised the study. All authors reviewed and commented on the manuscript.

\section{References}

1. Zaki AM, van Boheemen S, Bestebroer TM, Osterhaus $A D$, Fouchier RA. Isolation of a novel coronavirus from a man with pneumonia in Saudi Arabia. N Engl J Med. 
2012;367(19):1814-20.

http://dx.doi.org/10.1056/NEJMoa1211721

PMid:23075143

2. World Health Organization (WHO). Middle East respiratory syndrome coronavirus (MERS-CoV) summary and literature update - as of 22 November 2013. Geneva: WHO. [Accessed 10 Dec 2013]. Available from: http://www.who.int/csr/disease/ coronavirus_infections/Update12_MERSCoV_update_22Nov13. pdf

3. The Who Mers-Cov Research Group. State of Knowledge and Data Gaps of Middle East Respiratory Syndrome Coronavirus (MERS-CoV) in Humans. PLoS Curr. 2013;5.

PubMed PMID: 24270606.

4. Assiri A, McGeer A, Perl TM, Price CS, Al Rabeeah

AA, Cummings DA, et al. Hospital outbreak of Middle East respiratory syndrome coronavirus. N Engl J Med. 2013;369(5):407-16.

http://dx.doi.org/10.1056/NEJMoa1306742

PMid:23782161

5. Cotten M, Watson SJ, Kellam P, Al-Rabeeah AA, Makhdoom HQ, Assiri A, et al.Transmission and evolution of the Middle East respiratory syndrome coronavirus in Saudi Arabia: a descriptive genomic study. Lancet. 2013 Sep 19. http://dx.doi.org/10.1016/S0140-6736(13)61887-5

6. Ithete NL, Stoffberg S, Corman VM, Cottontail VM, Richards LR, Schoeman MC, et al. Close relative of human Middle East respiratory syndrome coronavirus in bat, South Africa. Emerg Infect Dis. 2013 Oct;19(10):1697-9.

http://dx.doi.org/10.3201/eid1910.130946

PMid:24050621 PMCid:PMC3810765

7. Memish ZA, Mishra N, Olival KJ, Fagbo SF, Kapoor V, Epstein JH, et al. Middle East respiratory syndrome coronavirus in bats, saudi arabia. Emerg Infect Dis. 2013;19(11). http://dx.doi.org/10.3201/eid1911.131172 PMid:24206838 PMCid:PMC3837665

8. Reusken CB, Haagmans BL, Müller MA, Gutierrez C, Godeke GJ, Meyer B, et al. Middle East respiratory syndrome coronavirus neutralising serum antibodies in dromedary camels: a comparative serological study. Lancet Infect Dis. 2013;13(10):859-66. http://dx.doi.org/10.1016/S1473-3099(13)70164-6

9. Perera RA, Wang P, Gomaa MR, El-Shesheny R, Kandeil A, Bagato O, et al. Seroepidemiology for MERS coronavirus using microneutralisation and pseudoparticle virus neutralisation assays reveal a high prevalence of antibody in dromedary camels in Egypt, June 2013. Euro Surveill. 2013;18(36): pii=20574. Available from: http://www. eurosurveillance.org/ViewArticle.aspx?Articleld=20574 PMid:24079378

10. Flajnik MF, Deschacht N, Muyldermans S. A case of convergence: why did a simple alternative to canonical antibodies arise in sharks and camels? PLoS Biol. 2011;9(8):e1001120.

http://dx.doi.org/10.1371/journal.pbio.1001120 PMid:21829328 PMCid:PMC3149040 